\title{
Imaging human skin autograft integration with optical coherence tomography
}

\author{
Anthony J. Deegan ${ }^{1 \#}$, Jie Lu ${ }^{1 \#}$, Rajendra Sharma ${ }^{1}$, Samuel P. Mandell ${ }^{3}$, Ruikang K. Wang ${ }^{1,2} \wedge$ \\ ${ }^{1}$ Department of Bioengineering, University of Washington, Seattle, WA, USA; ${ }^{2}$ Department of Ophthalmology, University of Washington, Seattle, \\ WA, USA; ${ }^{3}$ Division of Trauma, Critical Care, and Burn, University of Washington, Harborview Medical Center, Seattle, WA, USA
}

\#These authors contributed equally to this work.

Correspondence to: Prof. Ruikang K. Wang. Department of Bioengineering, University of Washington, 3720 15th Ave. NE., Seattle, WA 98195, USA. Email: wangrk@uw.edu.

Background: Skin autografting is a common clinical procedure for reconstructive surgery. Despite its widespread use, very few studies have been conducted to non-invasively evaluate and monitor the vascular and structural features of skin grafts. This study, therefore, aims to demonstrate the potential of optical coherence tomography (OCT) alongside OCT-based angiography (OCTA) to non-invasively image and monitor human skin graft health and integration over time.

Methods: An in-house-built clinical prototype OCT system was used to acquire OCT/OCTA images from patients who underwent split-thickness skin graft surgery following severe burn damage to the skin. The OCT imaging was carried out at multiple locations over multiple time points with a field of view of $\sim 9 \mathrm{~mm}$ $\times 9 \mathrm{~mm}$ and a penetration depth of $\sim 1.5 \mathrm{~mm}$. In addition to obtaining high-resolution qualitative images, we also quantitatively measured and compared specific structural and vascular parameters, such as identifiable layer thickness and corresponding vascular area density and diameter.

Results: Two patients (patient \#1 and \#2) were enrolled for this preliminary study. Vascular and structural features were successfully imaged and measured in the graft tissue and integration layer immediately beneath at different time points. Revascularization, healing, and integration were monitored with patient-specific details. Results of the quantitative image analysis from patient \#1 indicated that integration layer thickness 16-day post-surgery was significantly less $(\sim 50 \%)$ than that of 7 -day post-surgery. Additionally, with patient \#2, significant growth $(20 \%)$ was seen with the vascular area density of both the graft and corresponding integration layer beneath between 6 and 14 days post-surgery.

Conclusions: Our preliminary studies show that OCT/OCTA has clinical potential to image and measure numerous features of human skin graft health and integration in the days and weeks following split-thickness surgery. For the first time, we demonstrate the applicability of non-invasive imaging technology for novel clinical uses that could eventually aid in the betterment of surgical practices and clinical outcomes.

Keywords: Optical coherence tomography angiography (OCTA); integration; split-thickness skin graft; vasculature; structure

Submitted Jun 10, 2020. Accepted for publication Sep 25, 2020.

doi: 10.21037 /qims-20-750

View this article at: http://dx.doi.org/10.21037/qims-20-750

\footnotetext{
^ ORCID: 0000-0001-5169-8822.
} 


\section{Introduction}

Skin autografting is a form of reconstructive surgery that involves the transfer of skin from a healthy donor site to a recipient site in a need of repair on the same person. It has been widely used as a treatment strategy to improve the quality of life for patients suffering from burn damage, trauma, or cancer (1). Skin autografts can be harvested as split-thickness or full-thickness grafts, of which splitthickness skin grafts are more frequently used for tissue coverage. A split-thickness skin graft includes epidermis and varying amounts of dermis, with a thickness ranging between 125 and $750 \mu \mathrm{m}$ (2). With that, any visualization of a skin graft integrating into the recipient tissue beneath would require an imaging technique capable of providing cellular-level resolution and an imaging depth of at least $1 \mathrm{~mm}$. Initially, a skin graft does not have its own blood supply and must therefore rely on the wound bed for nutrients (3). Such a process ensures that the viability of a skin graft during the early stages of incorporation is entirely determined by its integration with the blood supply of the recipient tissue beneath. Any buildup of fluid between the skin graft and wound bed caused by hematoma, seroma or infection could impact the ability of the recipient tissue's blood supply to connect with the graft resulting in an incomplete graft take or even failure. Therefore, a technique to reliably assess the structure and vascular perfusion of individual layers, i.e., the graft itself, referred to here as the graft layer, and the intermediate layer between the graft and recipient tissue, referred to here as the integration layer, over time would greatly facilitate our ability to identify complications early and monitor the integration process of the skin graft.

Whilst numerous studies have been carried out to evaluate and optimize both surgical and post-operative practices regarding skin transplantation (4-12), little research has been conducted to develop appropriate techniques to objectively and accurately evaluate skin graft health post-surgery. The studies that have assessed graft healing were typically invasive or employed the use of animal models $(6,9,12)$, or measured superficial parameters, such as graft contraction (10), or re-epithelialization (11). Notwithstanding the considerable improvements made in the availability and applicability of modern, non-invasive imaging technologies (13), the imaging and assessment of human skin autograft subsurface structure and vasculature during the integration process remains underexplored and underutilized.
In a clinical setting, graft health is typically assessed via visual inspection by health providers (14). In the event of graft failure or incomplete integration, the extraction of biopsies is currently thought to be the only accurate method available to determine the cause of failure. This is not ideal, however, as biopsies cause further tissue damage, can be time consuming, increase the risk of infection, and may introduce inaccurate results if the wrong site is chosen for assessment. Moreover, repeated biopsies could result in a complication of scarring. The need for a non-invasive imaging modality that could aid in monitoring graft health is ever-present. For that reason, we have employed here the use of a non-invasive imaging modality capable of assessing both vascular and structural features of the skin to monitor graft health over time.

Optical coherence tomography (OCT) with its cellular level resolution, imaging depth of $\sim 1.5 \mathrm{~mm}$ and threedimensional (3D) imaging capabilities is a potential tool to non-invasively image and monitor the integration of the skin graft in real time. It uses infrared light in a similar way to how ultrasonography utilizes sound waves to image subsurface tissue structures (15). With improved resolution, sensitivity and scanning speeds, OCT is quickly becoming an important tool for both research and clinical practices in dermatology (16-18). OCT-based angiography (OCTA) is a functional extension of OCT that can provide additional information beyond that of tissue structure, such as identifying functional blood vessels. OCTA distinguishes blood vessels from the surrounding static tissue by measuring OCT signal differences at the same location over multiple time points in quick succession. Blood vessels become distinct because the OCT signal derived from blood flow varies over time, while the OCT signal backscattered from the surrounding static tissue remains almost unchanged $(19,20)$. Additionally, advances in the development of algorithms, such as attenuation correction, segmentation, en face mapping, thickness measurement, and vascular measurement, allow for the imaging and analyzing of structural and vascular features in individual layers both qualitatively and quantitatively.

In this study, we aimed to explore the potential of applying OCT/OCTA to visualize and monitor the healing and integration of skin autografts. To do this, we used a prototype OCT imaging system capable of extracting OCTA information to visualize and analyze the vascular (via OCTA-derived blood vessel mapping) and structural (via OCT-derived attenuation correction mapping) features 


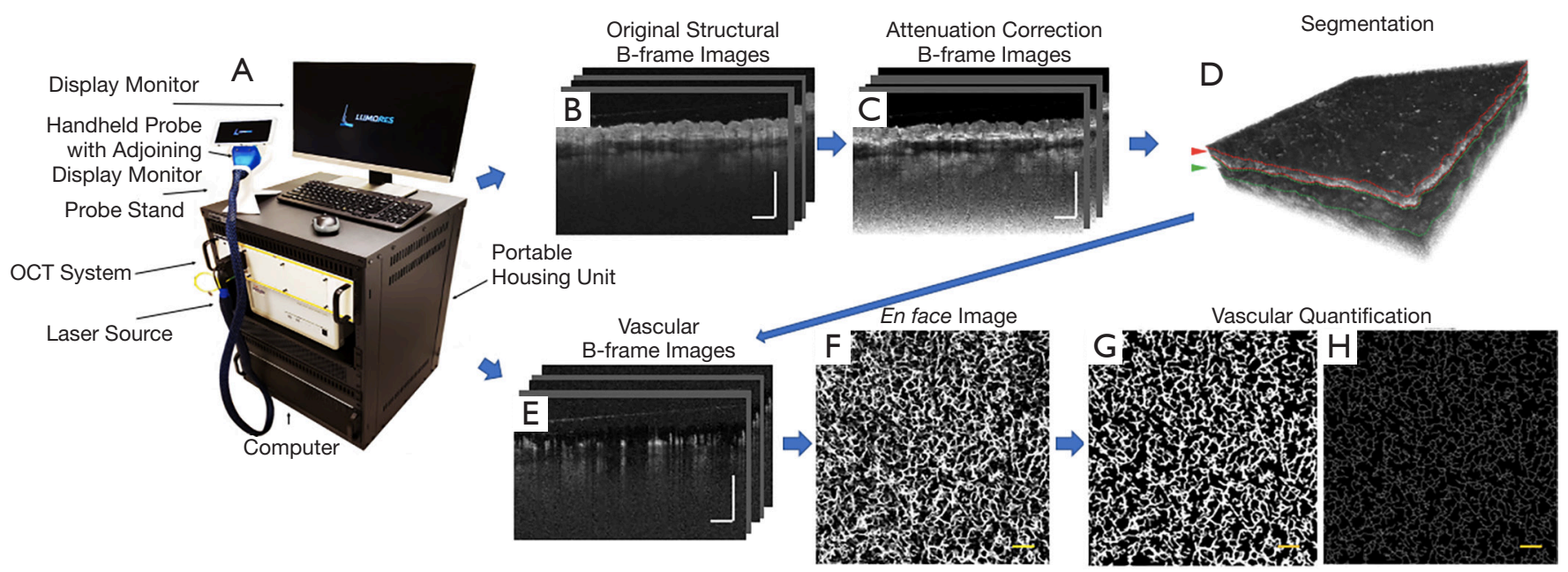

Figure 1 Images showing the prototype OCT system alongside a schematic of data processing. (A) The prototype OCT system capable of OCTA data acquisition; (B) original structural cross-section B-frame images; (C) attenuation correction cross-section B-frame images; (D) 3D structure image. Red lines highlight graft layer boundaries (graft layer further highlighted with a red arrow) and green lines highlight integration layer boundaries (integration layer further highlighted with a green arrow). Layer segmentation was carried out using these lines; (E) vascular cross-section B-frame images; (F) original en face projection of 3D blood vessels; $(\mathrm{G})$ binarized vascular image used for the quantification of vascular area density; $(\mathrm{H})$ skeletonized vascular image used for the quantification of vascular diameter. Scale bar represents $1 \mathrm{~mm}$.

of the graft itself and the corresponding integration layer beneath.

\section{Methods}

\section{Subject volunteers}

Two patients (denoted as patient \#1 and patient \#2) who underwent split-thickness skin graft surgery following severe burn damage to the skin were recruited, consented, and scanned at two separate time points post-operatively. Both patients had $250 \mu \mathrm{m}$-thick autologous grafts excised from the anterior of the femoral thigh and transplanted to the dorsum (patient \#1) or ventrum (patient \#2) of the right forearm. Both patients received sheet grafts (not meshed). Patient \#1 was scanned at 7 and 16 days post-surgery, and patient \#2 was scanned at 6 and 14 days post-surgery. Multiple scan sites, i.e., 3-4, were chosen for each patient. For comparative purposes, patient \#1 had adjacent burn site and control site scans (contralesional forearm) carried out in addition to three graft site scans. Patient \#2 had only three graft scans carried out. The use of OCT laboratory equipment on human subjects was reviewed and approved by the Institutional Review Board of the University of Washington.

\section{OCT imaging system}

An in-house-built clinical prototype OCT system (Figure $1 A$ ) was used in this study. The system was similar to that published in $(21,22)$ but re-configured for clinical research in dermatology. Briefly, the system was equipped with a $200 \mathrm{kHz}$ swept laser source (SL1310V1-10048, Thorlabs Inc.) with a central wavelength of $1,310 \mathrm{~nm}$ (infrared range) and spectral bandwidth of $100 \mathrm{~nm}$ to provide an axial resolution of $\sim 8 \mu \mathrm{m}$ in tissue $(\sim 11 \mu \mathrm{m}$ in air). The sample arm was configured as a hand-held probe with a $6.5 ", 1080 \mathrm{p}$ display monitor, sample spacer, and disposable contact unit. A $5 \times$ objective lens focused the light source into a beam spot with an incident power of $5 \mathrm{~mW}$, whilst a paired galvo scanner was used to scan the probe beam over the skin to form raster sampling patterns consisting of fast ( $\mathrm{x}$-axis) and slow (y-axis) axes.

\section{Imaging protocol}

Briefly, 3D OCT scans were acquired with a field of view of $9 \mathrm{~mm} \times 9 \mathrm{~mm}$, a penetration depth of $\sim 1.5 \mathrm{~mm}$ and lateral resolution of $\sim 10 \mu \mathrm{m}$ using 800 A-lines to produce a single B-frame, and $800 \mathrm{~B}$-frame locations with 4 repeated $\mathrm{B}$-frames in each, to produce a single volumetric C-scan. 
Each of the 4 repeated, single-location B-frames were then registered into one using optical microangiography (OMAG) (23-25) to extrapolate OCTA information, i.e., blood flow information (Figure 1). OCT (Figure 1B) and OCTA (Figure $1 E$ ) provided structural and vascular information, respectively. The total acquisition time for one imaging session, including preparation time and all scans, was approximately $\sim 30$ minutes with each $3 \mathrm{D}$ scan taking $\sim 6$ seconds.

\section{Attenuation correction mapping}

The contrast between the graft and integration layer is low in the OCT images because the intensity of OCT light is exponentially attenuated along its path as the light beam propagates through the skin due to the scattering and absorption of said light. To enhance the contrast between the graft layer and integration layer in the OCT image, so as to facilitate the segmentation algorithm for accurately separating adjacent layers, we applied an attenuation correction algorithm to the structural images. According to Vermeer et al. (26), the OCT signals compensated with attenuation correction at each pixel, $\mu(i)$ can be expressed as:

$$
\mu[i] \approx \frac{I[i]}{2 \Delta \sum_{i+1}^{N} I[i]}
$$

Where $I(i)$ refers to the OCT signal at the $i$-th pixel along the depth, $\Delta$ can be adjusted based on specific tissue characteristics, and $\mathrm{N}$ is the last pixel of the A-line. It is assumed that most light is already completely attenuated at the end of each A-line.

Pixel intensity exponentiation is a common method used to enhance imaging contrast, but it is not always feasible in areas with large attenuation since processed signals could present with more shadows or signal loss (27). The attenuation correction algorithm above could restore signal in attenuated areas. Therefore, to further improve the contrast of the image, we applied exponentiation after attenuation compensation, and each pixel intensity is converted as:

$$
U_{\text {Attn-corr }}[i]=\frac{I^{2}[i]}{2 \sum_{i+1}^{N} I^{2}[i]}
$$

Where $U_{\text {Attn-corr }}$ is the signal corrected by attenuation compensation and pixel intensity exponentiation. This approach improves the ability to detect the boundaries between adjacent layers (Figure 1C), which aids us in segmentation.

\section{Image segmentation}

As mentioned above, assessing the structure and vasculature of the graft and integration layers independently plays a critical role in identifying complications early and evaluating the integration of said skin graft. To visualize the individual layer's features independently, we segmented the $3 \mathrm{D}$ attenuation correction images into separate graft and integration layers. Each layer was acquired by semiautomatically outlining the layer boundaries within the attenuation correction B-frame images of each volumetric C-scan (28) (Figure 1D). The same segmentation information was then applied to vascular imaging volumes; that is, the vascular imaging volumes were also segmented at the same depths (Figure 1E).

\section{En face mapping of layer thickness and vessel measures}

For convenient visualization and further quantification, en face images were generated based on segmented volume information. An en face thickness map was obtained by calculating the depth separation between the upper and lower boundaries of each layer (i.e., graft layer and integration layer) at each A-line. A color-code was subsequently applied to represent a thickness range of 0-500 $\mu \mathrm{m}$. The processed OCTA volumetric data was collapsed into a two-dimensional (2D) map with a maximum intensity projection (MIP), where the highest intensity along the depth was chosen for each A-line, allowing for the generation of a 2D map, termed as en face map (Figure $1 F$ ). Where applicable, color codes were applied to en face-projected vasculature to represent a vessel depth ranging from $0-1 \mathrm{~mm}$.

\section{Quantification of layer thickness, and vascular area density and diameter}

To demonstrate the potential of OCT to assess postoperative recovery of skin grafting objectively, we measured multiple layer thicknesses, as well as vascular area density and diameter values from both the graft and corresponding integration layer of both patients at indicated time points. Mean thickness measurements were taken from each layer (i.e., graft layer and integration layer) based on the segmentation information. Measurements of vascular area density and vascular diameter were performed on each layer using en face images using a procedure developed during previous studies $(29,30)$, where vascular area 


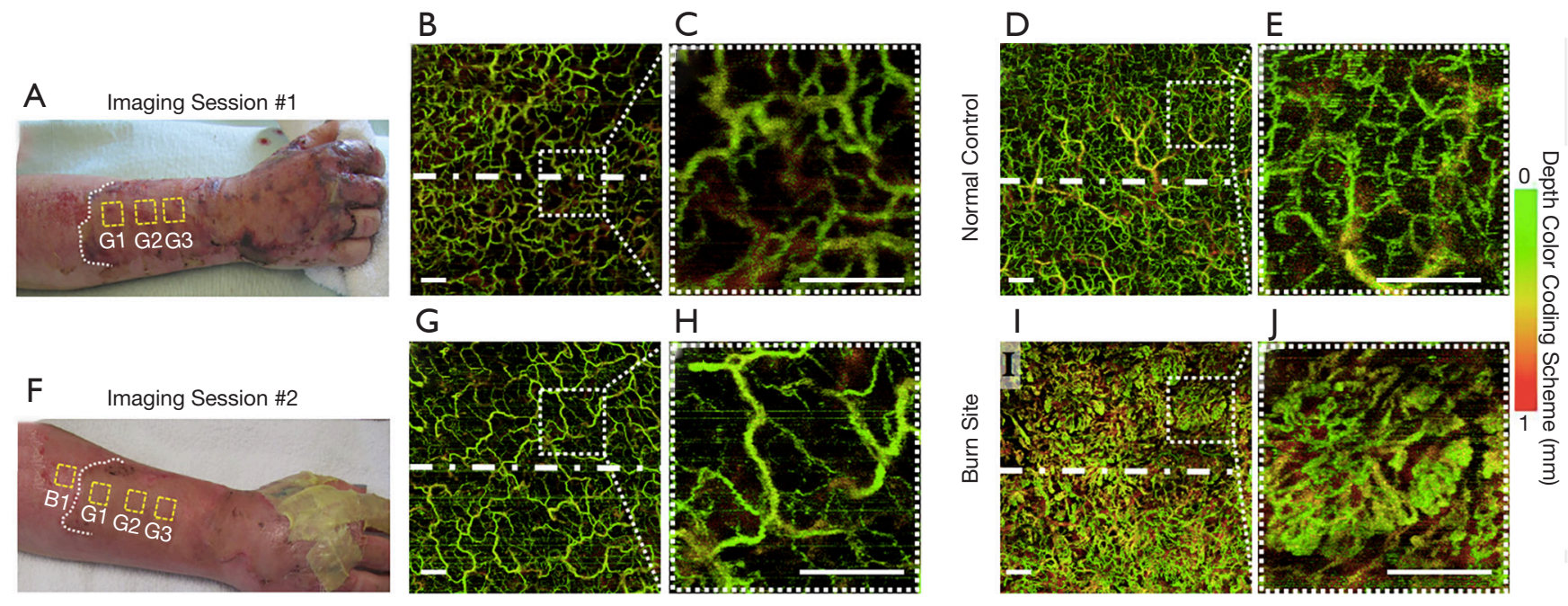

Figure 2 En face OCTA imaging of patient \#1. (A,F) showing photographs of the grafted forearm 7 and 16 days post-surgery, respectively. Highlighted are approximate scan sites (yellow perforated boxes) labeled G1, G2, G3 and B1. G1-G3 refer to three graft sites, and B1 refers to a burn site adjacent to the graft. The graft boundary is also highlighted (white perforated line). $(B, C)$ demonstrating the graft vasculature 7 days post-surgery from scan site $G 2$. (D,E) showing the vasculature of the normal skin (contralesional forearm) on the same patient. $(\mathrm{G}, \mathrm{H})$ showing the graft vasculature 16 days post-surgery from scan site G2. (I,J) showing the vasculature of a burn site adjacent to the graft 23 days after the initially burn injury from scan site B1. (C,E,H,J) Magnified images of the regions highlighted in the dashed rectangles shown in (B,D,G,I). All en face images are maximum intensity projected representing the depth of $0-1 \mathrm{~mm}$. Color bar represents the vessel depth. Scale bar represents $1 \mathrm{~mm}$. Shown are the three segmented slabs representing the depths of (A,B,C,D,E): 265-530 $\mu \mathrm{m}$ (papillary dermis).

density refers to the percentage of vascular area in OCTA images. To calculate vascular area density, the en face vascular map was processed into a binary image (Figure $1 G$ ) after carrying out global thresholding, and applying a hessian filter and adaptive thresholding. A skeletonized vascular image (Figure $1 \mathrm{H}$ ) was then generated from the binary image, in which each blood vessel, ignoring its diameter, was shown as a single-pixel line. The length of the blood vessels was then extracted from the skeletonized image, which allowed us to calculate the average vascular diameter by dividing total vascular area by total vascular length.

\section{Statistical analysis}

Three measurements of each layer thickness, vascular area density and diameter were averaged and presented as mean \pm standard deviation. Both imaging sessions were compared using two sample $t$-tests. Statistical significance is represented at two levels: ${ }^{*} \mathrm{P} \leq 0.05$, and ${ }^{* *} \mathrm{P} \leq 0.01$.

\section{Results}

Presented here are the OCT- and OCTA-derived features observed over time as the grafted autologous skin tissue integrated with the severely burn-damaged skin tissue beneath. Figure 2 shows the vascular (through en face vessel maps) changes that occurred within and immediately below the graft over the dorsum of the right forearm of patient \#1. Figure $2 A$ and $F$ shows the grafted forearm at 7 and 16 days post-surgery, respectively, with approximate scan locations and dividing graft borderline highlighted. For comparative purposes, multiple OCT scans were taken of the graft, adjacent burn and equivalent healthy sites (contralesional forearm). Figure $2 D$ alongside a magnified inset, Figure $2 E$, shows that the control site has a homogenous vascular morphology, density, and distribution. Comparatively, Figure $2 I$ and $\mathcal{F}$ (a burn site adjacent to the graft) show that the burn-damaged skin has extremely high vascular area density, even at 23 days post-burn, making it difficult to identify individual vessel morphology. Comparatively again, Figure $2 B$ and $C$ show that the vasculature of the graft just 7 days post-surgery 

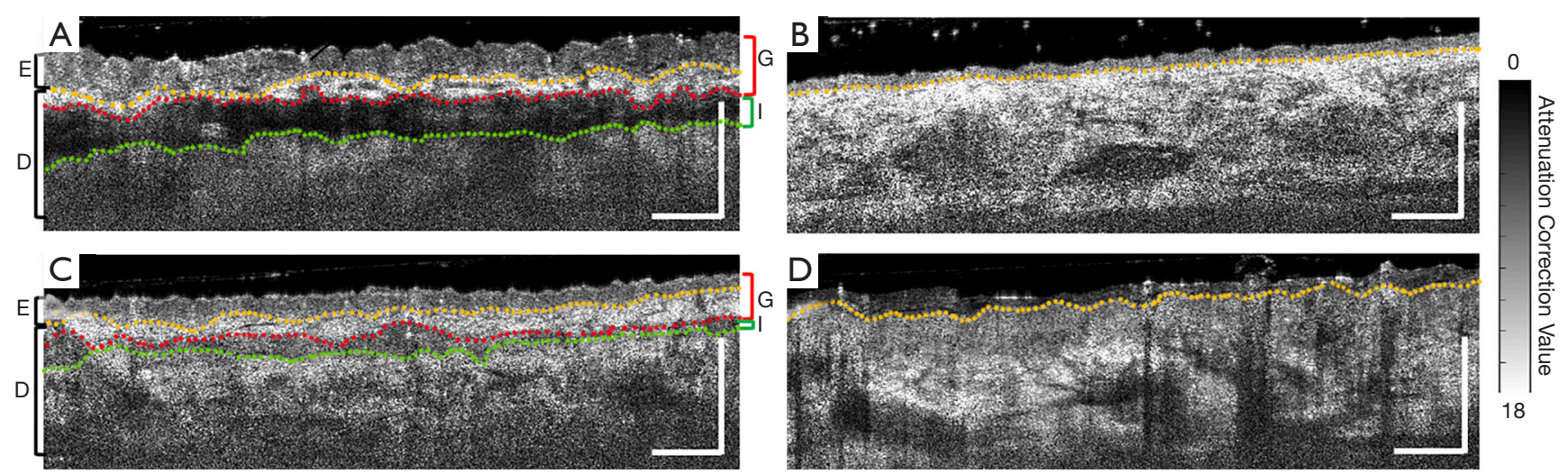

Figure 3 Representative cross-sectional B-frames of OCT structure (after attenuation correction processing). Shown are the B-frames acquired at the site indicated by the white dashed lines in Figure 2A,C,E,G. (A) Shows the graft 7 days postsurgery; (B) shows the normal skin (contralesional forearm) on the same patient; (C) shows the graft 16 days post-surgery; (D) shows a burn site adjacent to the graft 23 days after the initial burn injury. Yellow dashed lines highlight the epidermal-dermal junction. Red dashed lines highlight the boundary between graft layer and the integration layer. Green dashed lines highlight the boundary between the integration layer and the recipient tissue. Scale bar represents $1 \mathrm{~mm}$. E, epidermis; D, dermis; G, graft layer; and I, integration layer. Color bar represents OCT intensity after attenuation correction.

already with a morphology and distribution more akin to that of the normal control (Figure 2D,E), albeit with fewer superficial blood vessels (shown as green vessels in Figure 2). Figure $2 \mathrm{G}$ alongside a magnified inset, Figure $2 \mathrm{H}$, shows the vasculature of the graft 16 days post-surgery, where the vasculature appears even more reminiscent of the normal control compared to the first imaging session.

Figure 3 shows the representative cross-sectional B-frames of the tissue structure (after attenuation correction processing) at the position indicated by the white dashed lines in Figure 2. In these images, the epidermal-dermal junction and adjacent layer boundaries are clearly visible. The yellow dashed line highlights the epidermal-dermal junction. The red dashed line highlights the lower boundary of the graft layer and the upper boundary of the integration layer. The green dashed line highlights the lower boundary of the integration layer and the upper boundary of the recipient tissue. Figure $3 B$ shows the normal control with a smooth and consistent structure. In contrast, Figure $3 D$ shows the burn-damaged skin structure with a rough epidermis. Figure $3 A$ demonstrates that the epidermis of the graft tissue is significantly thicker than that of the normal control site with the integration layer also being clearly visible beneath. Figure $3 C$ shows that over time, the epidermal thickness returned to a level closer to that of the normal control, as evidenced when comparing the second imaging session to the first. Additionally, the integration layer in the second imaging session is also thinner and less pronounced compared to the first imaging session.

Shown in Figure 4 are the same graft scans presented in Figure 2 and Figure 3 only segmented into two distinct layers, i.e., the graft layer and integration layer, to separately demonstrate the vasculature and thickness maps of each layer. For patient \#1, it appears that the vascular distributions in both the graft and integration layer were more homogeneous in the second imaging session compared to the first. Additionally, the graft and integration layers both seemed to have thinned between the first and second imaging session.

Figure 5 shows the vascular (through en face vascular maps) and structural (through representative cross-sectional B-frames of attenuation correction maps) changes that occurred within and immediately below the graft over the ventrum of the right forearm of patient \#2. Figure $5 A$ and $E$ shows the grafted forearm at 6 and 14 days post-surgery, respectively, with approximate scan locations highlighted. Figure $5 B$ alongside a magnified inset, Figure $5 C$, shows the grafted skin 6 days post-surgery with a sparse density of blood vessels. Clearly visible is a gap between the graft tissue and recipient tissue in the cross-sectional B-frames (Figure 5D). Figure $5 F$ and $G$ shows that the distribution of blood flow 14-day post-surgery is more homogenous compared to the blood flow of 6-day post-surgery. Figure $5 H$ shows that the integration layer is now 


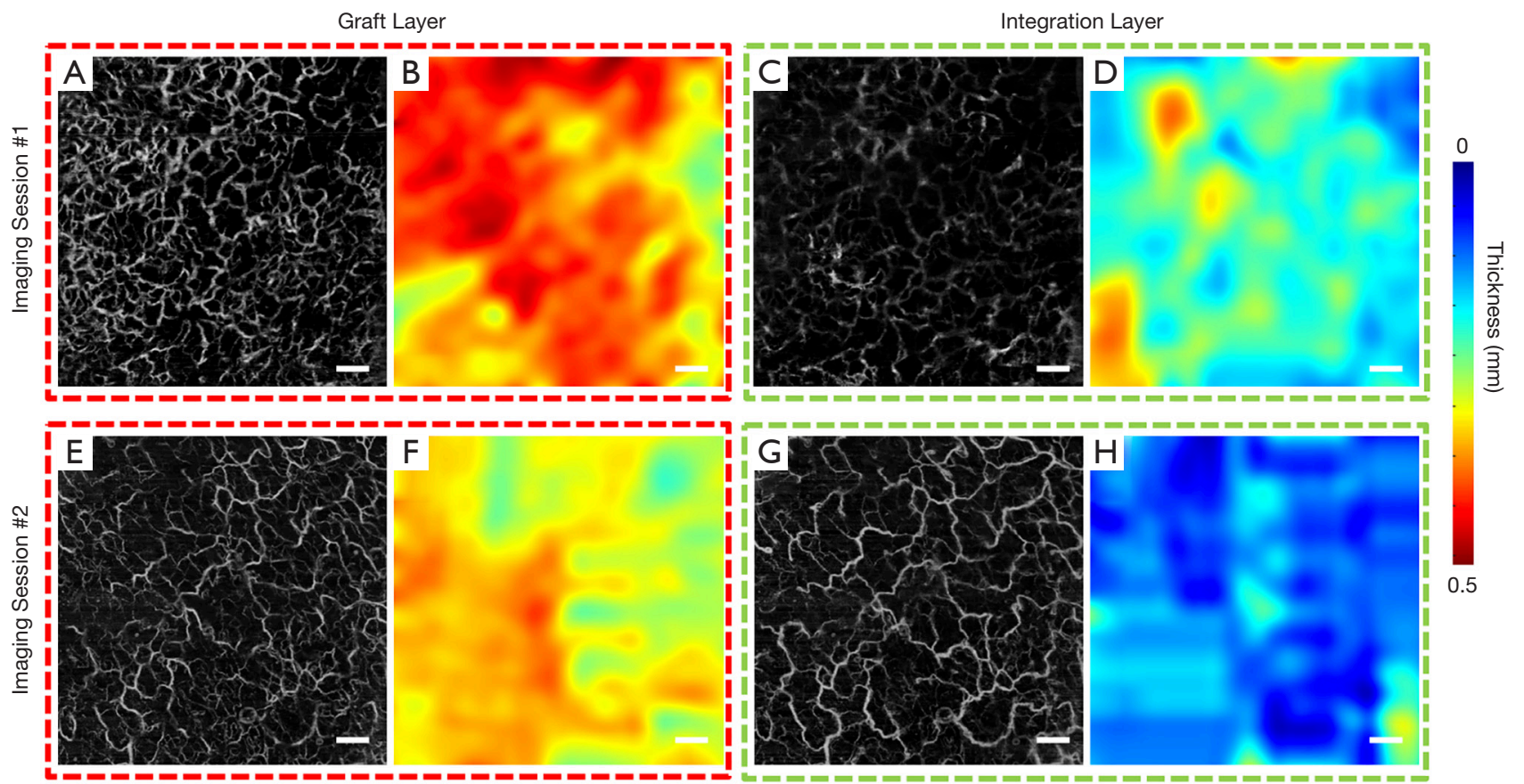

Figure 4 En face projected blood vessel and layer thickness maps from patient \#1 spanning two imaging sessions. (A,C,E,G) En face projected vasculature maps derived from the graft and integration layers, respectively. (B,D,F,H) Thickness maps derived from the graft and integration layers, respectively. All en face blood vessel images are maximum intensity projected. Color bar presents a depth range of $0-0.5 \mathrm{~mm}$. Scale bar represents $1 \mathrm{~mm}$.

significantly less obvious in the second imaging session compared to the first. Moreover, Figure $5 F$ presents dark vessel-like structures which are highlighted by the yellow arrow.

Shown in Figure 6 are the same graft scans presented in Figure 5 segmented into two layers, i.e., the graft and integration layers, to separately demonstrate the vasculature and thickness maps of each layer. For patient \#2, blood vessels were denser in the second imaging session compared to the first for both the graft and integration layers. However, neither the graft nor the integration layer appeared to have changed significantly in terms of thickness between the first and second imaging sessions.

Table 1 shows the detailed quantification results of layer thickness measurements, and vascular area density and diameter measurements from both patients at both time points. Integration layer thickness at the second time point was significantly lower than that of the first time point with patient \#1 ( $\mathrm{P}=0.0047)$. Significant growth can be seen for both graft vascular area density $(\mathrm{P}=0.042)$ as well as integration layer vascular area density $(\mathrm{P}=0.0052)$ between both imaging sessions for patient \#2. Vascular diameter within the integration layer of patient \#2 also increased significantly between the first and second imaging sessions $(\mathrm{P}=0.042)$.

\section{Discussion and conclusions}

Skin autografting has been clinically used worldwide as a treatment strategy for numerous conditions including burns, soft tissue wounds, and cancer. However, there is still a failure rate of $10-30 \%$ (31). Few studies have been conducted to monitor the wound healing, integration and maturation process of a skin graft. Therefore, the need for an accurate, non-invasive imaging tool to evaluate graft health and integration remains. To the best of our knowledge, the work presented in this study demonstrates for the very first time the ability of OCT and OCTA to image and monitor the health and integration of autologous human skin grafts following split thickness skin graft surgery. Two patients were imaged separately post-surgery at two time points. Alterations to the graft tissue itself and the tissue immediately beneath the graft tissue, referred to here as the integration layer, were monitored through 


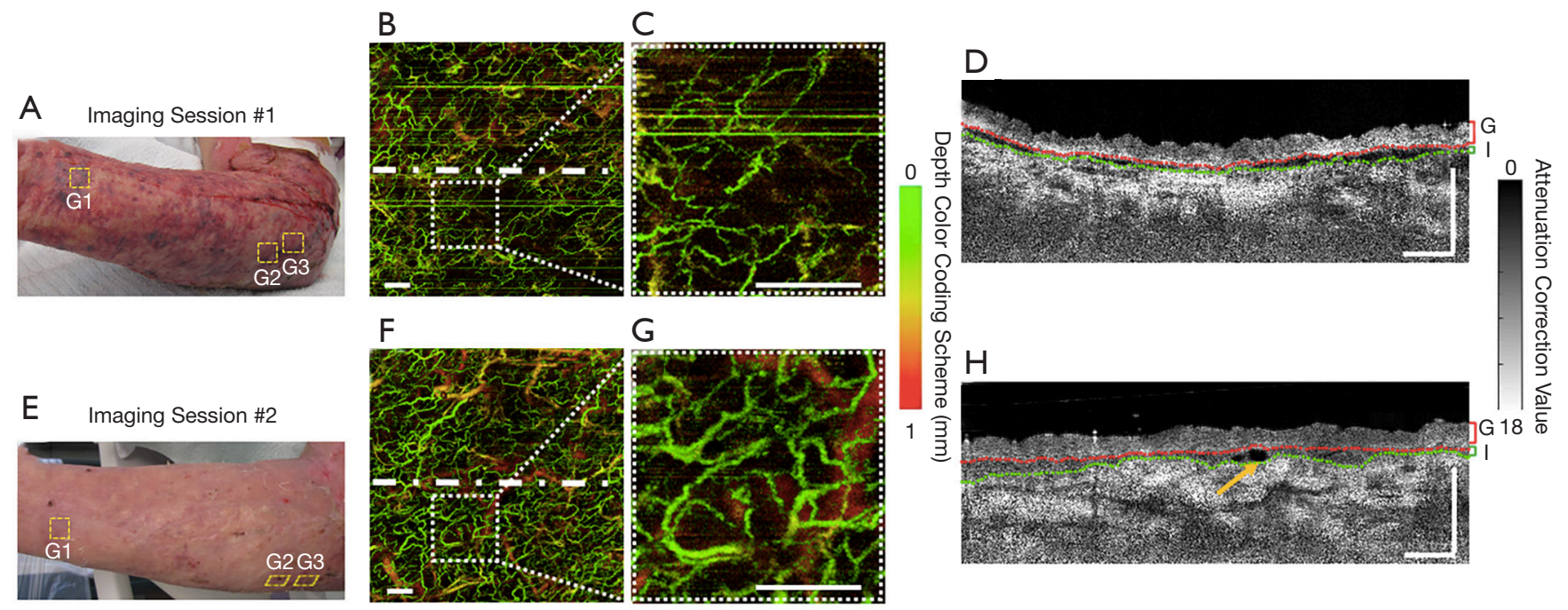

Figure 5 En face OCTA images and structural (after attenuation correction processing) cross-sectional B-frames from patient \#2. (A,E) showing photographs of the grafted forearm 6 days and 14 days post-surgery, respectively. Highlighted are three scan sites (red perforated boxes) labeled G1, G2, G3. G1-G3 refer to three graft sites. (B,C,D) showing the graft 6 days postsurgery from scan site G2. (F,G,H) showing the graft 14 days post-surgery from scan site G2. (B,C,F,G) En face projected blood vessel maps with color coded vessel depth. $(\mathrm{C}, \mathrm{G})$ Magnified images of the regions highlighted in the dashed rectangles in $(\mathrm{B}, \mathrm{F}) .(\mathrm{D}, \mathrm{H})$ Representative cross-sectional B-frames of OCT structure corresponding to the white dashed line in $(\mathrm{B}, \mathrm{F})$. All en face images are maximum intensity projected representing the depth of $0-1 \mathrm{~mm}$. Color bar on the left represents vessel depth. Color bar on the right represents OCT intensity after attenuation correction. Scale bar represents $1 \mathrm{~mm}$. Yellow arrow: dark vessel-like structures. E, epidermis; D, dermis; G, graft layer; and I, integration layer.

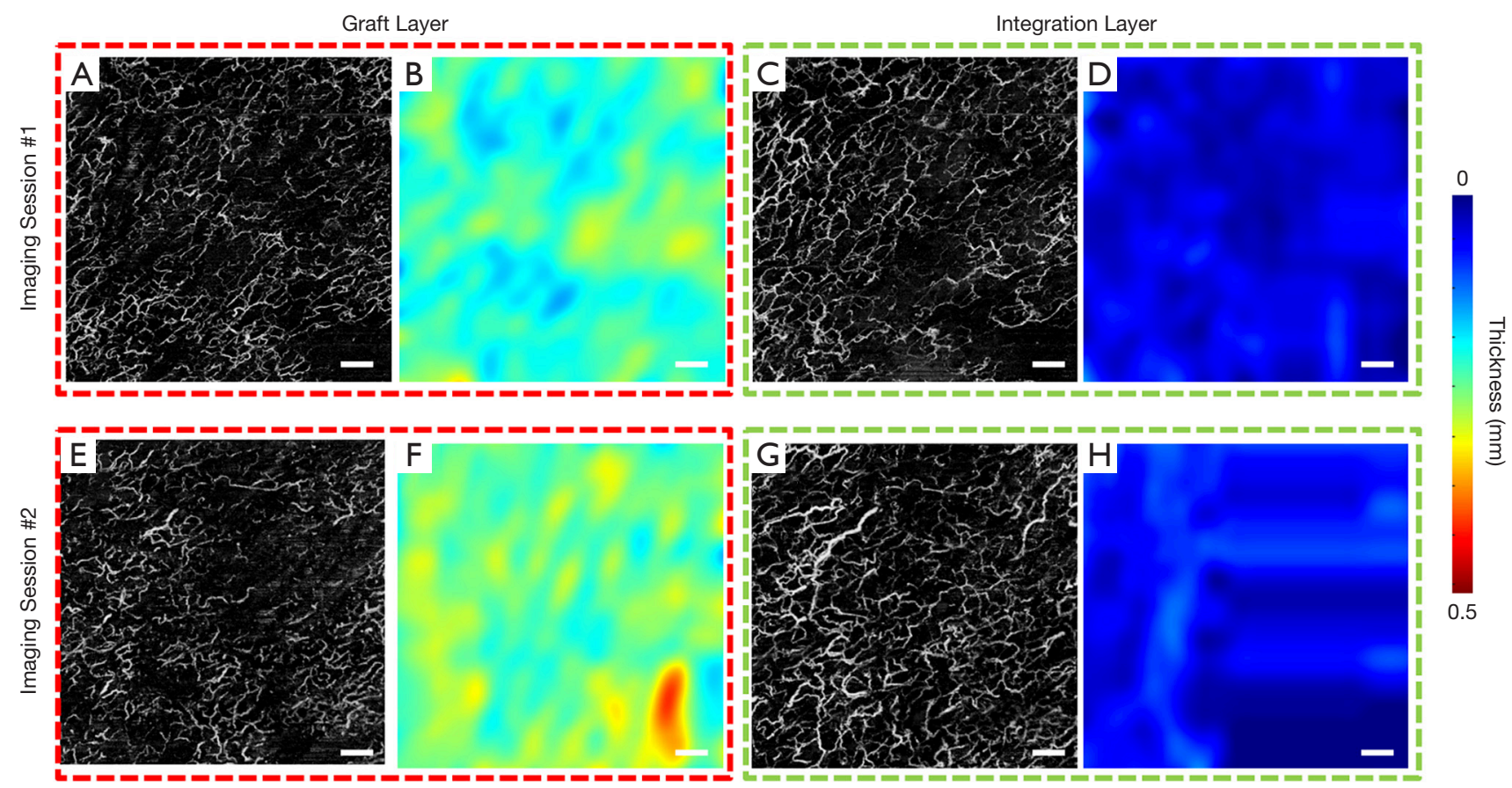

Figure 6 En face projected blood vessel and layer thickness maps of patient \#2 spanning two imaging sessions. (A,C,E,G) En face projected blood vessel maps derived from the graft and integration layers, respectively. (B,D,F,H) Thickness maps are derived from the graft and integration layers, respectively. All en face blood vessel images are maximum intensity projected. Color bar presents a depth range of $0-0.5 \mathrm{~mm}$. Scale bar represents $1 \mathrm{~mm}$. 
Table 1 Quantitative analysis of layer thickness and vascular parameter measurements

\begin{tabular}{|c|c|c|c|}
\hline Groups & Session \#1 $(N=3)$, mean $\pm S D$ & Session \#2 $(N=3)$, mean $\pm S D$ & $P$ value \\
\hline Graft layer thickness $(\mu \mathrm{m})$ & $364.52 \pm 38.48$ & $335.00 \pm 42.50$ & 0.64 \\
\hline Integration layer thickness $(\mu \mathrm{m})$ & $235.53 \pm 17.88$ & $114.82 \pm 11.36$ & $0.0047^{\star \star}$ \\
\hline Graft layer vessel area density & $0.3609 \pm 0.0166$ & $0.3699 \pm 0.0274$ & 0.79 \\
\hline Graft layer vascular diameter $(\mu \mathrm{m})$ & $37.04 \pm 2.48$ & $37.28 \pm 1.32$ & 0.94 \\
\hline Integration layer vascular diameter $(\mu \mathrm{m})$ & $35.43 \pm 1.63$ & $39.66 \pm 2.14$ & 0.20 \\
\hline \multicolumn{4}{|l|}{ Patient \#2 } \\
\hline Graft layer thickness $(\mu \mathrm{m})$ & $285.11 \pm 41.18$ & $311.31 \pm 20.40$ & 0.61 \\
\hline Integration layer vessel area density & $0.2841 \pm 0.0079$ & $0.3470 \pm 0.0063$ & $0.0052^{\star \star}$ \\
\hline Graft layer vascular diameter $(\mu \mathrm{m})$ & $31.43 \pm 1.87$ & $36.07 \pm 1.32$ & 0.11 \\
\hline Integration layer vascular diameter $(\mu \mathrm{m})$ & $30.54 \pm 2.56$ & $39.09 \pm 1.27$ & $0.042^{*}$ \\
\hline
\end{tabular}

$\mathrm{P}$ values were obtained by two sample $t$-tests. * $\mathrm{P} \leq 0.05 ;{ }^{* \star}, \mathrm{P} \leq 0.01$.

the vascular (via blood vessel mapping) and structural (via attenuation correction mapping) features of both tissue layers.

The importance of graft revascularization for graft survival has been noted previously (32); accordingly, we aimed to utilize a non-invasive imaging modality capable of assessing skin features for evaluating graft health. As mentioned above, OCTA detects functional blood vessels by identifying differences between moving particles in the blood, i.e. red blood cells, and the surrounding static tissue. That is, OCTA would not detect the flow if there is no blood flow in the blood vessels or the blood flow is too slow that is below its detection limit (typically less than $\sim 10 \mu \mathrm{m} / \mathrm{s}$ ). A skin graft initially does not have its own blood supply; therefore, theoretically we should not observe any blood flow immediately after surgery. Blood flow was observed in the graft and integration layers from both patients at the first imaging session, which indicates that patient \#1 and patient \#2 had realized revascularization 7- and 6-day postsurgery, respectively. This is consistent with the theory that revascularization takes 3-7 days after surgery (33). This also proves the ability of OCT/OCTA to evaluate revascularization of a skin graft. There are two main hypotheses regarding the processes of the revascularization: (I) that graft vessels link to the existing wound tissue bed directly; and (II) that new vessels grow into the graft from the wound bed beneath, i.e., angiogenesis $(33,34)$. With the exact mechanism behind skin graft revascularization not yet being fully understood, OCT/OCTA could become an important tool to explore the mechanisms and dynamics of early vessel transformation. In addition, significant growth was observed both in graft vascular area density $(\mathrm{P}=0.042)$ and integration layer vascular area density $(\mathrm{P}=0.0052)$ between the two imaging points from patient \#2, indicating further revascularization of skin grafts over time.

Visually, epidermal thickening, a common feature of a graft with edema (35), was evident in patient \#1 as seen in the graft cross-sectional B-frame images (Figure $3 A$ ), which partially subsided later (Figure 3C). The edema is thought to result from lymphatic dysfunction (36) and inflammation. Lymphangiogenesis later contributes to the edema reduction (32). Dark vessel-like structures were visualized during the second imaging session for patient \#2 (Figure 5H). Patient \#1 too showed dark-vessel structures in both the graft layer and integration layer during the second imaging session, but for patient \#1, these structures were not as evident as patient \#2. These structures are thought to be lymphatic vessels, because lymph fluid is almost transparent, allowing lymphatic vessels to appear as dark regions (low scattering intensity) with vessel-like shapes in 
the OCT images. In this way, OCT may be used to provide evidences of lymphangiogenesis in terms of direct structural images and edema reduction.

Whilst both patients imaged offered an informative glimpse into the vascular and structural features of integrating graft tissue that could potentially allow a clinician or researcher to monitor the various stages of healing, an additional observation is unveiled when we compared data derived from patient \#1 with those derived from patient \#2. Taking both time points into account, patient \#1 had thicker epidermis that lead authors to believe that edema was still present 7 -day post-surgery, which then partially subsided 16-day post-surgery. Epidermal thickness for patient \#2, however, was notably more stable 6-day postsurgery, as evidenced by the similar measures taken 14-day post-surgery. For patient \#1, the thickness of the integration layer was significantly $(\sim 50 \%)$ reduced in the second imaging session compared to the first $(\mathrm{P}=0.0047)$, whilst patient \#2 did not due to an already thinned integration layer during first time imaging session. This may be due to several complications that arose in the days initially following surgery for patient \#1, which meant additional blebbing was required; thus, maintaining patient \#1 in an inflamed condition for longer than patient \#2.

This prolonged inflammation for patient \#1 is also thought to have affected vascular area density, as well as integration layer thickness. Simply, the aforementioned complications and consequent inflammation experienced by patient \#1 led to an increased vascular area density during imaging session \#1. As healing progressed for this patient, the increased vascular area density caused by inflammation during imaging session \#1 was replaced by an equally high vascular area density resulting from revascularization during imaging session \#2. Comparatively, patient \#2 did not experience the same degree of inflammation and so had a relatively low vascular area density during imaging session \#1 compared to patient \#1. As healing progressed for this patient also, revascularization led to a significantly higher vascular area density during imaging session $\# 2$. Whilst this might appear initially as though both patients revascularized at different rates, the values seen here are simply due to inflammation affecting the first of two time points. During imaging session \#2, both patients had again simply normalized. This additional observation highlights the advantages of using non-invasive imaging technology over other invasive modalities; in that, repeated measures can be conducted more easily over time without interfering with the healing process. This allows for a more in-depth assessment of healing.

Overall, these data, although limited, do offer an insight into the vascular and structural features observable via OCT and OCTA that may be useful to a clinician in establishing the integration and maturation progress of grafted skin in the days and weeks following surgery. Additionally, from a clinical standpoint, the potential applications for OCT and OCTA in graft imaging are vast. OCTA, for example, could not only be used to monitor graft integration but to also evaluate donor site vascularity prior to excision and aid with predicting graft survivability. OCTA also shows its potential in evaluating wound healing in burn site. For example, we suspect the vessels shown in Figure 27 are fenestrated vessels surrounding a hair follicle providing the necessary nutrients to accommodate re-epithelialization. This could be argued as a marker for skin healing if identified early enough as this would be a subsurface feature that would predate visible surface healing. The limited number of other non-invasive methods described in the literature (11) are restricted to assessing specific features of the graft alone and cannot assess donor site, integration layer, wound bed tissue, or incomplete graft intake. Even from a preclinical standpoint, OCT and OCTA can be employed for the assessment of grafting practices and engineered skin tissue substitutes-a growing area of research $(37,38)$. Polarization-sensitive OCT (PS-OCT), which is sensitive enough to detect different dermal layers as well as anisotropic biological structures $(39,40)$, e.g., collagenan important dermis component, and its restoration during wound healing process, would further enhance the use of OCT in skin autograft studies.

Several limitations in this study need to be acknowledged. Firstly, the field of view in this study is limited to $9 \mathrm{~mm} \times$ $9 \mathrm{~mm}$, which results in the loss of information outside the field of view. To minimize this bias, we scanned multiple locations over the graft of each patient in the hope of gathering as much information as possible. To further address this issue in future studies, some technologies with a wider field of view, such as laser speckle contrast imaging, could be used to objectively guide OCT imaging to the graft sites of interest. Secondly, our study is limited by a small cohort of subjects. It is worthwhile to mention that this study aims to explore the potential of applying OCT/OCTA to visualize and monitor the healing and integration of skin autografts, rather than provide novel medical discoveries. Certainly, to fully understand skin graft in OCT imaging, a large-scale clinical study would need to be conducted in the future. Thirdly, the penetration depth of OCT is limited 
to approximately $1.5 \mathrm{~mm}$, which could result in the loss of pertinent, deep structural and vascular information. This is a limitation of OCT in general and not an issue with our specific imaging system. With that, to overcome such a limitation, the use of a complimentary modality could again be used. Photoacoustic imaging (PAI) is an example of another non-invasive optical imaging modality with a potential penetration depth of $\sim 5 \mathrm{~mm}$. With a lateral resolution of $\sim 50 \mu \mathrm{m}$ and an axial resolution of $\sim 22 \mu \mathrm{m}$, however, the level of detail acquired with such would be considerably lower than OCT/OCTA, which has a lateral resolution of $\sim 10 \mu \mathrm{m}$ and an axial resolution of $\sim 8 \mu \mathrm{m}$ (41). This is not thought to be an issue though, as the vessels beyond several millimeters in depth would be significantly larger than those of more superficial depths, and would, therefore, not require the capillary-level detail afforded by OCT/OCTA. The combination of OCT/OCTA and PAI, therefore, could be a very promising route of investigation with both technologies working synergistically to solve the issue of penetration depth whilst maintaining a high level of detail where needed in skin graft evaluation.

In summary, OCT/OCTA in this study has shown its potential to image and measure numerous features of human skin graft health and integration in the days and weeks following split-thickness surgery. For the first time, we demonstrate the applicability of non-invasive OCT/ OCTA imaging technology for novel clinical uses that could aid in the betterment of surgical practices and clinical outcomes in the future.

\section{Acknowledgments}

Funding: This study was supported in part by Washington Research Foundation, and Research to Prevent Blindness. The funding organization had no role in the design or conduct of this research.

\section{Footnote}

Conflicts of Interest: All authors have completed the ICMJE uniform disclosure form (available at http:// dx.doi.org/10.21037/qims-20-750). RKW serves as an unpaid editorial board member of Quantitative Imaging in Medicine and Surgery. The authors have made the following financial disclosures: RKW discloses intellectual property owned by the Oregon Health and Science University and the University of Washington. RKW also receives research support from Carl Zeiss Meditec Inc., Moptim
Inc., Shiseido Company, Colgate Palmolive Company and Facebook technologies LLC. RKW is a consultant to Carl Zeiss Meditec. The other authors have no conflicts of interest to declare.

Ethical Statement: The subject imaging followed protocols reviewed and approved by the Institutional Review Board of Medical Sciences Subcommittee at the University of Washington, Seattle. The tenets of the Declaration of Helsinki and Health Insurance Portability and Accountability Act were followed. Informed consent forms were obtained from all subjects before participation.

Open Access Statement: This is an Open Access article distributed in accordance with the Creative Commons Attribution-NonCommercial-NoDerivs 4.0 International License (CC BY-NC-ND 4.0), which permits the noncommercial replication and distribution of the article with the strict proviso that no changes or edits are made and the original work is properly cited (including links to both the formal publication through the relevant DOI and the license). See: https://creativecommons.org/licenses/by-nc-nd/4.0/.

\section{References}

1. Orgill DP. Excision and skin grafting of thermal burns. $\mathrm{N}$ Engl J Med 2009;360:893-901.

2. Johnson TM, Ratner D, Nelson BR. Soft tissue reconstruction with skin grafting. J Am Acad Dermatol 1992;27:151-65.

3. Ratner D. Skin grafting. Semin Cutan Med Surg 2003;22:295-305.

4. Achora S, Muliira JK, Thanka AN. Strategies to promote healing of split thickness skin grafts: an integrative review. Review J Wound Ostomy Continence Nurs 2014;41:3359; quiz E1-2.

5. Carvalho JC, Palero JA, Jurna M. Real-time imaging of suction blistering in human skin using optical coherence tomography. Biomed Opt Express 2015;6:4790-5.

6. Dhillon M, Carter CP, Morrison J, Hislop WS, Currie WJ. A comparison of skin graft success in the head \& neck with and without the use of a pressure dressing. J Maxillofac Oral Surg 2015;14:240-2.

7. Erel E, Sinha M, Nancarrow JD. The 'pull out' tie-over dressing. J Plast Reconstr Aesthet Surg 2008;61:460-1.

8. Knapik A, Kornmann K, Kerl K, Calcagni M, Contaldo C, Vollmar B, Giovanoli P, Lindenblatt N. Practice of split-thickness skin graft storage and histological 
assessment of tissue quality. J Plast Reconstr Aesthet Surg 2013;66:827-34.

9. Zdichavsky M, Jones JW, Ustuner ET, Ren X, Edelstein J, Maldonado C, Breidenbach W, Gruber SA, Ray M, Barker JH. Scoring of skin rejection in a swine composite tissue allograft model. J Surg Res 1999;85:1-8.

10. Stekelenburg CM, Simons JM, Tuinebreijer WE, van Zuijlen PP. Analyzing contraction of full thickness skin grafts in time: Choosing the donor site does matter. Burns 2016;42:1471-6.

11. Hauser J, Lehnhardt M, Daigeler A, Langer S, Steinau HU, Vogt PM. Photoplanimetric evaluation and impedance measurement of split-thickness skin grafts: a new model for objective wound-healing assessment in clinical trials. Skin Res Technol 2009;15:168-71.

12. Gould DJ, Reece GP. Skin graft vascular maturation and remodeling: a multifractal approach to morphological quantification. Microcirculation 2012;19:652-63.

13. Deegan AJ, Wang RK. Microvascular imaging of the skin. Phys Med Biol 2019;64:07TR1.

14. Timar-Banu $\mathrm{O}$, Beauregard H, Tousignant J, Lassonde M, Harris P, Viau G, Vachon L, Levy E, Abribat T. Development of noninvasive and quantitative methodologies for the assessment of chronic ulcers and scars in humans. Wound Repair Regen 2001;9:123-32.

15. Tomlins PH, Wang RK. Theory, developments and applications of optical coherence tomography. J Phys D Appl Phys 2005;38:2519-35.

16. Deegan AJ, Talebi-Liasi F, Song S, Li Y, Xu J, Men S, Shinohara MM, Flowers ME, Lee SJ, Wang RK. Optical coherence tomography angiography of normal skin and inflammatory dermatologic conditions. Lasers Surg Med 2018;50:183-93.

17. Deegan AJ, Wang W, Men S, Li Y, Song S, Xu J, Wang RK. Optical coherence tomography angiography monitors human cutaneous wound healing over time. Quant Imaging Med Surg 2018;8:135-50.

18. Olsen J, Holmes J, Jemec GB. Advances in optical coherence tomography in dermatology-a review. J Biomed Opt 2018;23:1-10.

19. Chen CL, Wang RK. Optical coherence tomography based angiography [Invited]. Biomed Opt Express 2017;8:1056-82.

20. Wang RK, Jacques SL, Ma Z, Hurst S, Hanson SR, Gruber A. Three dimensional optical angiography. Opt Express 2007;15:4083-97.

21. Song S, Zhou K, Xu JJ, Zhang Q, Lyu S, Wang R. Development of a clinical prototype of a miniature hand- held optical coherence tomography probe for prematurity and pediatric ophthalmic imaging. Biomed Opt Express 2019;10:2383-98.

22. Xu J, Song S, Men S, Wang RK. Long ranging swept-source optical coherence tomographybased angiography outperforms its spectral-domain counterpart in imaging human skin microcirculations. J Biomed Opt 2017;22:1-11.

23. Zhang Q, Wang J, Wang RK. Highly efficient eigen decomposition based statistical optical microangiography. Quant Imaging Med Surg 2016;6:557-63.

24. Wang RK, An L, Francis P, Wilson DJ. Depth-resolved imaging of capillary networks in retina and choroid using ultrahigh sensitive optical microangiography. Opt Lett 2010;35:1467-9.

25. Yousefi S, Zhi Z, Wang RK. Eigendecompositionbased clutter filtering technique for optical microangiography. IEEE Trans Biomed Eng 2011;58:10.1109/ TBME.2011.2152839.

26. Vermeer KA, Mo J, Weda JJ, Lemij HG, de Boer JF. Depth-resolved model-based reconstruction of attenuation coefficients in optical coherence tomography. Biomed Opt Express 2013;5:322-37.

27. Zhou H, Chu Z, Zhang Q, Dai Y, Gregori G, Rosenfeld PJ, Wang RK. Attenuation correction assisted automatic segmentation for assessing choroidal thickness and vasculature with swept-source OCT. Biomed Opt Express 2018;9:6067-80.

28. Yin X, Chao JR, Wang RK. User-guided segmentation for volumetric retinal optical coherence tomography images. J Biomed Opt 2014;19:086020.

29. Reif R, Qin J, An L, Zhi Z, Dziennis S, Wang R. Quantifying optical microangiography images obtained from a spectral domain optical coherence tomography system. Int J Biomed Imaging 2012;2012:509783.

30. Chu Z, Lin J, Gao C, Xin C, Zhang Q, Chen CL, Roisman L, Gregori G, Rosenfeld PJ, Wang RK. Quantitative assessment of the retinal microvasculature using optical coherence tomography angiography. J Biomed Opt 2016;21:66008.

31. Braza ME and Fahrenkopf MP. Split-Thickness Skin Grafts. Treasure Island (FL): StatPearls Publishing, 2020. Available online: https://www.ncbi.nlm.nih.gov/books/ NBK551561/

32. Frueh FS, Sanchez-Macedo N, Calcagni M, Giovanoli P, Lindenblatt N. The Crucial Role of Vascularization and Lymphangiogenesis in Skin Reconstruction. Eur Surg Res 2018;59:242-54. 
33. Leung JJ, Fish J. Skin Grafts. University of Toronto Medical Journal 2009;84:135-50.

34. Lindenblatt N, Calcagni M, Contaldo C, Menger MD, Giovanoli P, Vollmar B. A new model for studying the revascularization of skin grafts in vivo: the role of angiogenesis. Plast Reconstr Surg 2008;122:1669-80.

35. Simman R, Phavixay L. Split-thickness skin grafts remain the gold standard for the closure of large acute and chronic wounds. J Am Col Certif Wound Spec 2011;3:55-9.

36. Norrmén C, Tammela T, Petrova TV, Alitalo K. Biological basis of therapeutic lymphangiogenesis. Circulation 2011;123:1335-51.

37. Hur GY, Seo DK, Lee JW. Contracture of skin graft in human burns: effect of artificial dermis. Burns 2014;40:1497-503.

Cite this article as: Deegan AJ, Lu J, Sharma R, Mandell SP, Wang RK. Imaging human skin autograft integration with optical coherence tomography. Quant Imaging Med Surg 2021;11(2):784-796. doi: 10.21037/qims-20-750
38. Marino D, Luginbuhl J, Scola S, Meuli M, Reichmann E. Bioengineering dermo-epidermal skin grafts with blood and lymphatic capillaries. Sci Transl Med 2014;6:221ra14.

39. Baumann B. Polarization Sensitive Optical Coherence Tomography: A Review of Technology and Applications. Appl Sci 2017;7:474.

40. Tang $\mathrm{P}, \mathrm{Xu} \mathrm{J}$, Wang RK. Imaging and visualization of the polarization state of the probing beam in polarizationsensitive optical coherence tomography. Applied Physics Letters 2018;113:231101.

41. Liu M, Drexler W. Optical coherence tomography angiography and photoacoustic imaging in dermatology. Photochem Photobiol Sci 2019;18:945-62. 